\title{
SOME FACTORS IN THE CONTROL OF POLIOMYELITIS*
}

\author{
BY \\ R. W. FAIRBROTHER, M.D., M.R.C.P. \\ LECTURER IN BACTERIOLOGY, UNIVERSITY OF MANCHESTER
}

(With Special Plate)

This country is particularly fortunate in that extensive epidemics of infantile paralysis have been extremely infrequent. Small outbreaks-frequently involving schools and institutions-have, however, been reported at various times, while an examination of the weekly notifications of infectious diseases indicates that sporadic cases are always present. It thus appears that poliomyelitis is a problem of some importance to the public health authorities.

In the event of an outbreak the physician is faced with three main problems:

1. The possibility of determining and limiting the extent of the outbreak-epidemiology.

2. The treatment of contacts, with regard both to general measures and to specific immunization-prophylaxis.

3. The value of specific serum as a therapeutic measure -therapy.

In order to appreciate the difficulties surmounting these questions it is essential to have some knowledge of the infecting agent and the manner in which the disease process develops. These points will be briefly considered before an attempt is made to answer the aforementioned questions.

\section{Aetiology and Pathogenesis}

The infecting agent is accepted by the majority of workers as a filterable virus. It is extremely minute, readily traversing bacterial filters, and invisible under the highest powers of the microscope. It cannot be cultivated by any of the standard bacteriological methods, and the only experimental animal in which the disease can be reproduced is the monkey.

The spontaneous disease is encountered only in man. The experimental disease in the monkey is, however, very similar to the fatal human form, and is readily produced by intracerebral, intraneural, or intrathecal inoculation of a potent virus. The disease process cannot be studied in man, as the essential lesions are confined to the central nervous system, and consequently this problem has been investigated in the experimental condition.

The spread of the virus was considered to take place by the perineural lymphatics, from the nasopharynx to the cerebro-spinal fluid, from which it reached the nerve cells. Hurst and I, in 1929, propounded the theory of axonal spread-namely, that the spread of the virus from the nasopharynx to the anterior horn cells of the cord occurred through the brain tissue by way of the axis cylinders. The axonal theory has since received much support from work carried out in America. Faber and Gerhardt have also drawn attention to the important observation that the terminal processes of the olfactory nerves-the olfactory hairs-lie exposed to the air in the mucous membrane of the upper part of the nasal cavity. Thus in a portion of the nasal cavity there is a direct gateway to the central nervous system for the virus. The virus is strictly neurotropic, and the essential lesions are confined to the central nervous system. These consist primarily of vacuolation and degeneration of the nerve cells, with a secondary mononuclear infiltration, perivascular, diffuse, and meningeal (see Fig. 1 on Special Plate).

* Paper read before the Society of Medical Officers of Health North-Western Branch, January 11th, 1935.
The experimental condition almost invariably ends fatally, whereas in man the mortality rate, while subject to considerable variation, is definitely lower -5 to 20 per cent. While there is no doubt that the virulence and dosage of the virus are important factors in this distinction, another factor of great importance is the increased resistance offered to the virus by the human nerve cells as compared with those of the monkey.

\section{Epidemiology}

It is widely accepted at the present time that the portal of entry of the virus is the nasopharynx, and that the virus is conveyed from man to man by droplet infection. It has been stated that infection may be conveyed by drinking-water and milk, but conclusive proofs that such vehicles are of great importance have not been produced. Experimental results indicate very strongly that the portal of entry is in the nasopharynx, while the demonstration of the virus in the nasopharyngeal washings of cases and contacts provides important confirmatory evidence. From the data available it would appear that the virus on implantation in the nasopharynx gains entrance by the exposed olfactory hairs, and then passes along the olfactory nerve to the bulb, from which it disseminates through the mid-brain to the cord, attacking various nerve cells on its passage.

Paralysis, however, by no means invariably follows the implantation of the virus in the nasopharynx. It is highly probable that only a small percentage of infected individuals show muscular involvement. Statistical evidence and serological tests indicate that in the majority of cases subclinical infection follows-that is, the individual experiences no ill effect from the presence and activity of the virus, but apparently acquires an active immunity. Similar subclinical infections are quite common in other infectious diseases, as diphtheria and measles.

In other instances there is a marked reaction to the virus with the appearance of definite symptoms. These cases, found most commonly in children between the ages of 2 and 5 years, have been classified according to the nature of the clinical findings. It is sufficient to mention those most commonly encountered: (1) spinal form ; (2) bulbar form ; (3) abortive form.

In the first two forms muscular weakness and paralysis are found in the respective regions. In the last group are those cases showing pyrexia with vague nervous signs, as headache, and presenting definite changes in the cerebro-spinal fluid. These, however, recover without any obvious muscular involvement. This is probably a common sequence of events; Harmon (1934) collected figures illustrating the incidence of non-paralytic poliomyelitis, and found that approximately 70 per cent. of over 500 untreated cases showed no sign of paralysis. This is an important point in assessing the value of serum therapy on statistical lines, and a failure to appreciate it has probably been responsible for many of the extravagant claims for the value of serum therapy.

Two factors appear to be largely responsible for this variation in the individual response. The first is the virulence and dosage of the infecting virus. These doubtless vary considerably in the different epidemics. It is 
also highly probable that in the presence of an epidemic both the virulence and the dosage of the virus are likely to be greater than in inter-epidemic periods. The presence of different strains of virus has been suggested, but the evidence put forward to support this view is far from conclusive. The second factor in the individual variation of the disease is the resistance offered by the individual. The necessity of approximating the virus to the nervous system in order to produce infection has already been emphasized.

Once within the nervous system, the struggle for supremacy between the virus and the nerve cells takes place. The incubation period is uncertain, though it is probably longer than many consider. I suggest seven to ten days as the probable time, as the incubation period represents the time taken by the virus after implantation in the nasopharynx in reaching susceptible nerve cells. The preparalytic stage appears to be the phase at which the cells and the virus are battling for supremacy. Experimental evidence indicates that in the first instance only a proportion of the nerve cells of an infected region are attacked, and if these are able to withstand the attack of the virus recovery follows without muscular involvement. If, however, the virus is more active, more nerve cells will be attacked, and some will be permanently damaged. The extent of the damage will determine the degree of residual paralysis. In other cases, where the virus is particularly active, the disease progresses, the medulla is involved, and death supervenes.

The capacity for recovery exercised by the nerve cells is illustrated in Fig. 2 (see Special Plate).

This is a cross-section of the cervical enlargement of a monkey killed thirty-five days after the appearance of symptoms. This animal was one of the few that recovered after the inoculation of a potent virus ; eight days after intracerebral inoculation it exhibited a marked paresis of the right lower limb; twenty-four hours later both lower limbs and the eyelids showed marked paresis ; on the twelfth day the upper limbs became involved-the right arm was completely paralysed, while the left showed paresis. The animal was now prostrate, but instead of taking the usual course recovery took place, slow at first, but after seven to ten days a wide range of movement was obtained in all limbs except the right arm. This paralysis persisted in spite of treatment with ultra-violet light and massage. In order to obtain a picture of the cord in cases of recovery the animal was etherized and a detailed histological examination was carried out. In this section, which is at the segment corresponding to the paralysis, a marked reduction in the number of nerve cells on the paralysed side is observed; on the other side a more or less normal appearance is found.

In fatal cases the characteristic lesions are found in the cord, and virus can readily be demonstrated in the spinal cord, with less frequency in the nasopharyngeal washings, and sometimes in the brain.

We must next examine the reaction of the community to infection with the virus of poliomyelitis. The disease is usually encountered in children of school age, although in outbreaks occurring in rural districts adults are frequently involved. The explanation offered to account for the comparative immunity of adults is that in crowded or urban communities there is a greater chance of dissemination of the virus. Adults are therefore more likely to possess immunity following either previous subclinical infection or a definite attack. Young infants are not so frequently involved, as here the chance of infection is less, and in the first six months of life passive immunity is frequently present, due to the transplacental passage of maternal antibodies. In rural communities the possibility of a previous infection is reduced, and the individuals are more exposed to infection in the presence of an epidemic. People of all ages are also liable to infection in these districts.
In the event of an epidemic it is important to realize that only a small proportion of individuals harbouring the virus exhibit muscular impairment. Many experience abortive attacks-these, in the absence of frank cases of poliomyelitis, are extremely difficult to diagnose-and a number have merely subclinical infection. All these individuals are, however, carriers of the virus, and a potential danger to the community. Another point is that as the incubation period is comparatively long, many individuals will therefore have been disseminating the virus for days before the condition is notified. Attention should be paid to these points in dealing with an outbreak in a closed community such as a boarding school. The closure of the school and the subsequent dispersal of the scholars will lead to wide dissemination of the virus.

\section{Prophylaxis}

In the event of an epidemic prophylactic measures are of great importance; these may be conveniently considered as general and specific.

The general measures adopted should be first to isolate all definite or suspicious cases. A control of all contacts is desirable, and it is especially important to emphasize that contact with children should be avoided. The use of gargles has not been generally advocated. The main reason for this is based on a small experiment, which indicated that the nasopharyngeal mucosa possessed viricidal activity, and that this property was destroyed by antiseptics. Unfortunately this question has not been examined in detail, and as the virus has recently been demonstrated in nasopharyngeal washings of cases and contacts, this suggested viricidal activity does not appear to be very marked. A point not previously mentioned is that gargles would spread the virus in the nasopharynx, and so increase the possibility of it reaching the olfactory hairs which lie exposed in the upper part of the mucosa. Thus ordinary gargles cannot be recommended, but the use of oxidizing agents, such as hydrogen peroxide and potassium permanganate, appears advisable, for these are definitely injurious to the virus.

Specific measures involve the immunization of the individuals, either actively or passively. Active immunization, while highly satisfactory in the case of variola and rabies, is at the present time of no practical value in the prophylaxis of poliomyelitis. Killed virus has proved useless, and while immunity can be produced by the use of living virus, the difficulty of obtaining a dose which will invariably immunize without carrying any risk of producing the severe symptoms of the disease has not yet been overcome. This question has recently been receiving much attention, but although an advance has been made there is no doubt that the matter is still in the experimental stages.

Passive immunization by the intramuscular inoculation of a large dose of immune serum was advocated some time ago by Flexner. This procedure has since been adopted in combating several outbreaks, and the results appear to have been highly satisfactory. A statistical representation is of doubtful value, but several observers have reported a much greater incidence of cases in contacts not receiving serum than in those receiving it. At the present time the use of anti-poliomyelitis horse serum is recommended. This serum is readily obtained; the quantities available are thus greater than in the case of convalescent serum, and also its antiviral content is frequently higher than that of convalescent serum. It is, however, important to realize that the effects of passive immunization only last a few weeks, and should the epidemic persist a second inoculation after five to six weeks - following previous desensitization - should be carried out. 


\section{Therapy}

Many methods have been suggested to arrest and relieve paralysis : these again are best considered as general and specific.

General measures, such as adopted in the case of other infectious diseases-namely, rest in bed and attention to the diet-are very important therapeutic procedures, and should be enforced rigorously without delay. Numerous other measures have been tried, but the only one having definite favourable effects is spinal drainage. The corebrospinal fluid is usually under pressure, and shows a marked pleocytosis; the exudate in the cord tends to pass from the nervous tissue into the spinal fluid. Consequently, by repeated drainage it might be possible to alleviate the increased pressure in the cord by removing some of the infiltrating cells. This secondary infiltration, both perivascular and diffuse, is frequently well marked, and no doubt tends to exaggerate the symptoms.

Much controversy has arisen concerning the value of antiviral sera in the treatment of poliomyelitis. The use of convalescent serum in its treatment was first recommended by Netter in 1911. This was suggested by the fact that convalescent serum had been found, by means of the neutralization test, to possess antiviral bodies. (In this test an active virus is left in contact with the serum for a few hours, after which the mixture is inoculated intracerebrally into a monkey. If antiviral bodies are present the animal remains quite well; if not, experimental poliomyelitis develops. A control test in which. saline is substituted for the serum should always be carried out to check the potency of the virus.)

It thus appeared that an extremely valuable therapeutic agent was available. Unfortunately, many uncontrolled tests were made and untested serum was employed, and it is only in recent years that any useful contributions on the value of the serum have been made. The results obtained in the last few years have, however, been far from consistent. Some workers have obtained highly satisfactory results, while others have found no improvement to follow the administration of serum. This divergence of results is illustrated in the table.

Collected Results of Serum Therapy (Convalescent serum given in the early stages)

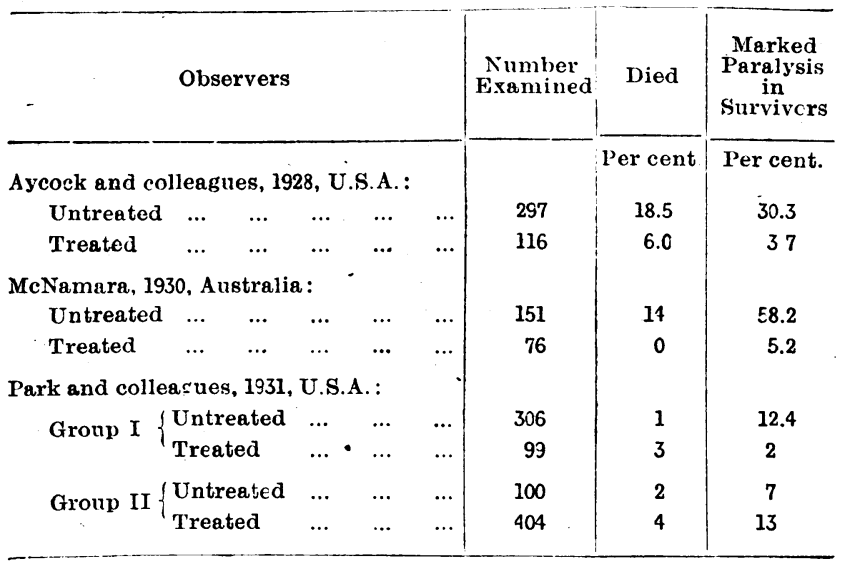

\section{The Use of Serum}

The question of serum therapy is obviously a matter of some complexity. In order to appreciate the difficulties of the question several points must be considered. The virus reaches the nervous tissue by way of the olfactory hairs in the nasopharynx, and thereafter is well protected from the serum; in many cases recovery takes place without paralysis (70 per cent., vide supra); epidemics vary in severity. These facts interfere considerably with the interpretation of the various statistical records of serum therapy. We have mentioned that in the preparalytic stage there was a struggle for supremacy between the nerve cells and the virus, that in the early stages only a proportion of the nerve cells in a given region are involved, and that some of the cells attacked may recover. The preparalytic phase, therefore, is obviously the optimum time to administer serum, as it is possible at this stage to reach certain cells before the virus, and in some cases the early administration of serum might throw the scales in favour of the cells. When, however, the virus held the upper hand serum might tend to limit the spread of the disease process to other regions of the cord, but it would have little effect on the primary focus. In the case of a heavy infection there appears to be little hope of benefit from the serum.

Therapeutic applications of serum in the experimental disease have proved useless. Thus dramatic recovery following the administration of serum is not likely to be obtained except probably in a few cases, some of which might equally have recovered without serum. As, however, other measures have not proved satisfactory-in fact, the majority have proved useless-I suggest that serum therapy should be tried whenever cases are diagnosed in the preparalytic phase. It is, however, important to emphasize that the serum should be given as early as possible-in the preparalytic stages-and that the statistical results may not be convincing. Harmon (1934), in an extensive review of the question of serum therapy, stated :

"Notwithstanding the total failure of statistical presentations to favour certain types of serum, clinical observations that have been almost universally made of rapid symptomatic response to the administration of serum by an immediate drop in temperature and marked improvement in symptoms cannot be totally disregarded."

It is important now to mention the types of serum available for the route of administration. Many sera have been suggested, but no serum should be employed which does not contain antiviral bodies. These bodies are demonstrated by the neutralization test in the monkey, and are usually found in convalescent serum, and also in the serum of many adults. In testing sera, in order to save expense, samples are pooled before examination. Horse serum, prepared by a long series of intramuscular injections of the virus, can also be used. As horses vary considerably in the ability to produce antibodies, careful titration experiments are necessary.

Various routes have been employed by different workers for the administration of the serum-intrathecal, intravenous, and intramuscular, either alone or in combination. It appears, however, that the results obtained after intravenous and intramuscular inoculation have been as satisfactory as those obtained by administration by other routes. These routes are also the less likely to give rise to reactions than the intrathecal. A study of the pathology of the condition also favours these routes. The virus is confined to the nervous tissue, and it is the aim to approximate the antibodies to the nerve cells. In the case of the cerebro-spinal fluid the flow is from the nerve tissue to the fluid, and, again, the virus has never been demonstrated in the fluid itself. These points do not offer support for the intrathecal injection of the serum.

Following intravenous or intramuscular inoculation the serum passes to the blood stream, and probably escapes into the nervous tissue by reason of the increased permeability of the vessels of the affected region. It thus appears advisable to give massive doses of serum by the intravenous or intramuscular route. 


\section{Conclusion}

The sporadic nature of the disease in this country suggests a wide dissemination of a virus of limited virulence, from which it follows that the majority of the adult population have acquired some immunity. This tends to contraindicate the appearance of an extensive epidemic. Small outbreaks in schools or institutions where children are collected are, however, always liable to appear. In handling these the various points $I$ have mentioned should be-enforced-namely, isolation of all cases or suspected cases, prophylactic inoculation of serum, and gargles of oxidizing substances for the contacts. In the treatment of cases diagnosed in the early stages serum therapy should be commenced without delay. In view of the crippling nature of the disease, aithough many cases will evidence little response, an improvement in a few cases is surely sufficient justification for serum therapy.

\section{CORNEAL GRAFTS WITH THE REPORT OF A CASE BY}

B. W. RYCROFT, M.D., D.O.M.S., F.R.C.S. HUNTERIAN PROFESSOR AND ASSISTANT SURGEON, ROYAL EYE HOSPITAL

AND

G. HANDELSMAN, L.M.S.S.A. SENIOR HOUSE-SURGEON, ROYAL EYE HOSPITAL, LONDON (With Special Plate)

Where blindness is entirely due to corneal opacity it has been proved that the operation of corneal grafting is of great value, and the recent investigations of Tudor Thomas have revived interest in a branch of surgery which was first successfully practised by von Hippel in 1888. From time to time the technique of such grafting has varied both in the shape of the graft and in the method of fixation.

On the one hand, Elschnig uses a circular trephine, whereas Castroviejo employs a twin-bladed knife which cuts a rectangular graft, and this he retains in position by means of a conjunctival flap. Tudor Thomas, basing his technique on several years of animal experimentation, obtains the graft by a circuiar trephine of 4 to $6 \mathrm{~mm}$. in diameter, arguing that this is much more likely to "take" than that which has corners or sharp angles. $\mathrm{He}$ retains the graft in position by overlay sutures stitched into the cornea on either side of the graft, and lays great stress on the necessity of fashioning a shelving bed for the reception of the graft. In order to allow of subsequent swelling of the graft, this is cut smaller than the bed into which it is to be placed, and in transference the graft is protected by means of sterile olive oil. In the following case an endeavour was made to simplify technique and to combine the trephine method with a complete conjunctival flap, such as is occasionally employed for the protection of large gaping corneal wounds.

Several criteria must be satisfied if the graft is to be successful ; they include complete sterility of the conjunctival sac, accurate projection of light, a pupillary aperture neither occluded nor secluded, and transparency of all ocular media other than that of the cornea. The tension of the eye must be within normal limits, and there must be no active inflammation. Cases which fulfil such conditions are: corneal opacification due to chemical burns, large healed ulcers, and interstitial keratitis which has been quiet for at least twelve months. The scars of old perforating ulcers in which the iris is adherent are not suitable, since, owing to this adhesion, there is a predisposition to vascularity, and consequent opacification of the graft. In our opinion the degree of vision should not be better than hand movements to justify what is still experimental surgery.

The following case is that of a woman who for many years had kerato-iritis of both eyes. Secondary glaucoma supervened in the right eye, which was removed, and complete opacification of the remaining eye had reduced the vision to perception of light. A corneal graft restored the vision to $6 / 24$, and allowed her to read ordinary manuscript.

\section{Case Record}

The patient was a married woman, aged 48. She attended the Royal Eye Hospital in July, 1920, and a diagnosis of tuberculous keratitis of the right eye was made. R.V., perception of light. The left eye at this time had fine corneal nebulae: L.V., 6/18. The usual local remedies, together with a course of tuberculin injections, were employed, and her atten Jance ceased in 1922 .

In 1923 the vision was the same in each eye, and in 1924 the right eye was removed on account of secondary glaucoma and an absence of perception of light. In April, 1930, the left eye developed acute kerato-iritis, which subsided after treatment, leaving a vision of $6 / 60$; at this time unit investigation was negative. In October, 1933, the vision was reduced to perception of light, and a blind certificate was issued.

In November, 1934, the patient came under our care, and it was decided to attempt keratoplasty. A complete investigation was carried out, and was negative apart from an increase of the blood sedimentation rate and evidence of old tuberculous cervical adenitis. The eye at this time showed grey infiltration of the whole of the cornea, with localized fasciculi of superficial conjunctival vessels and occasional evidence of deep vascular infiltration. It was not possible to discern the position of the pupil or iris pattern details either by diaphanoscopy or by direct slit-lamp investigation. The cornea was in the early stages of keratoglobus. Projection of light was accurate, and tension was $30 \mathrm{~mm}$. Schiötz.

Preliminary treatment began with four-hourly irrigations of oxycyanide of mercury lotion 1 in 8,000, and local ultra-violet radiation of three minutes' duration by full mercury vapour spectrum of the conjunctival fornices and entire cornea. Subsequent cultures were negative on three occasions. Miosis was obtained by constant instillation of guttae eserıne sulph., $1 / 2$ per cent. A donor became available in a case where a young man had sustained a penetrating injury of the upper lid and globe, which left the cornea intact but which disintegrated the posterior half of the eye.

\section{OPERATION}

The preparation of the site in the host and the enucleation of the donor's eye were commenced simultaneously. In the host a complete conjunctival flap was prepared circumscribing the limbus, and being separated well back to the equator of the globe. A purse-string suture, No. 1 black silk, was inserted in such a way as to render the aperture eccentric when tightened. Under 4 per cent. cocaine anaesthesia and with seventh nerve novocain block ( $\mathrm{O}^{\prime}$ Brien) a $4 \mathrm{~mm}$. circular bed was delineated slightly to the inner side of the centre of the cornea, so as to bring the proposed site of the graft below the margin of the upper lid. When the section had involved half the corneal thickness, the trephine was removed and the bed completed by scissors in such a manner as to produce a shelving edge towards the centre of the aperture. During this period the eye was constantly irrigated by normal saline at body temperature. When the corneal circle was removed the pupil could be seen firmly contracted, the centre of which coincided with the centre of the graft. This had been obtained purely by guesswork: Tudor Thomas has since elaborated a technique which enables the coincidence of the centre of the graft and the centre of the pupil to be accurately 


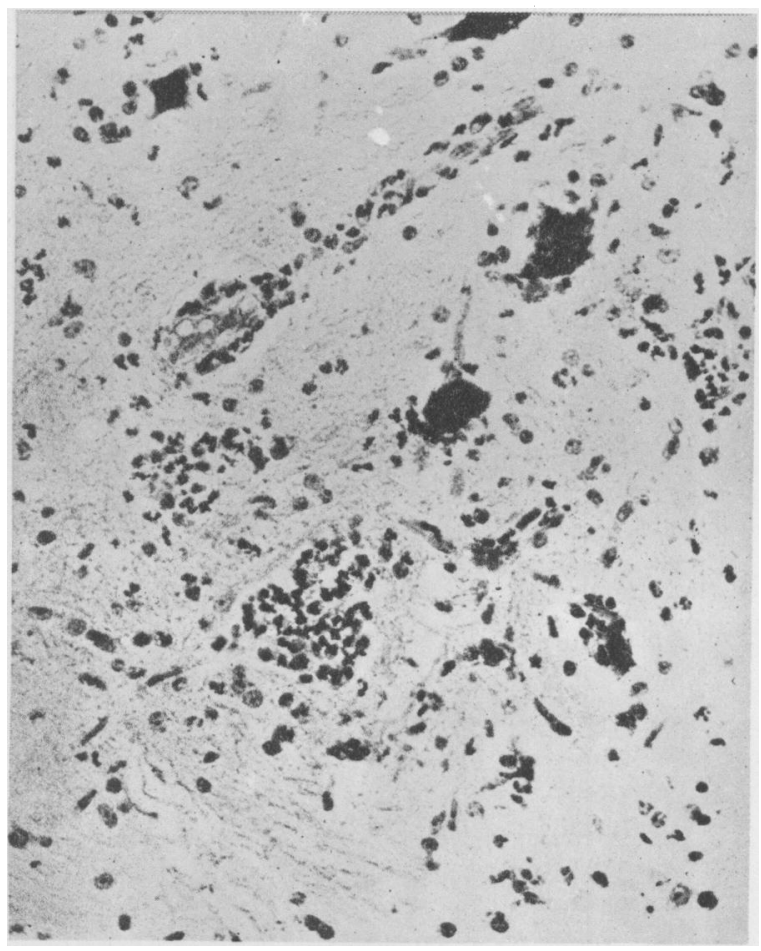

Fia. 1.-Experimental poliomyelitis. High-power photomicrograph of spinal cord, showing vacuolation, degeneration, and neuronophagia of the nerve cells.

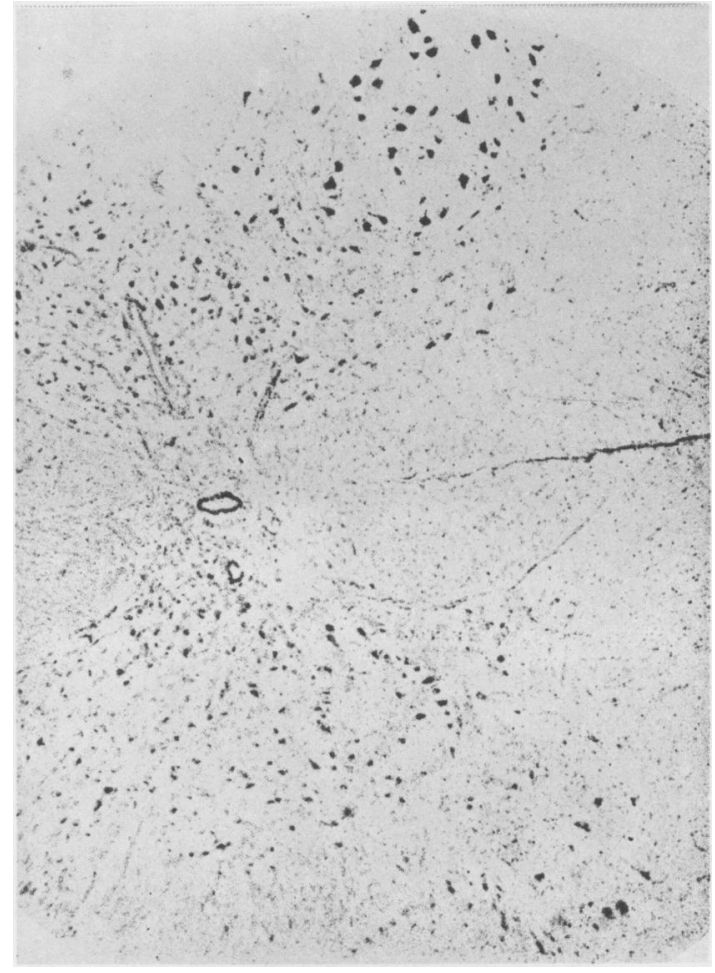

Fio. 2.-Experimental poliomyelitis. Iow-power photomlcrograph of spinal cord of recovered monkey with residual mlcrograph of spinal cord of recovered monkey with residual
paralysis. Note absence of large neurons in anterior horn of one side.

B. W. RYCROFT AND G. HANDELSMAN : CORNEAL GRAFTS

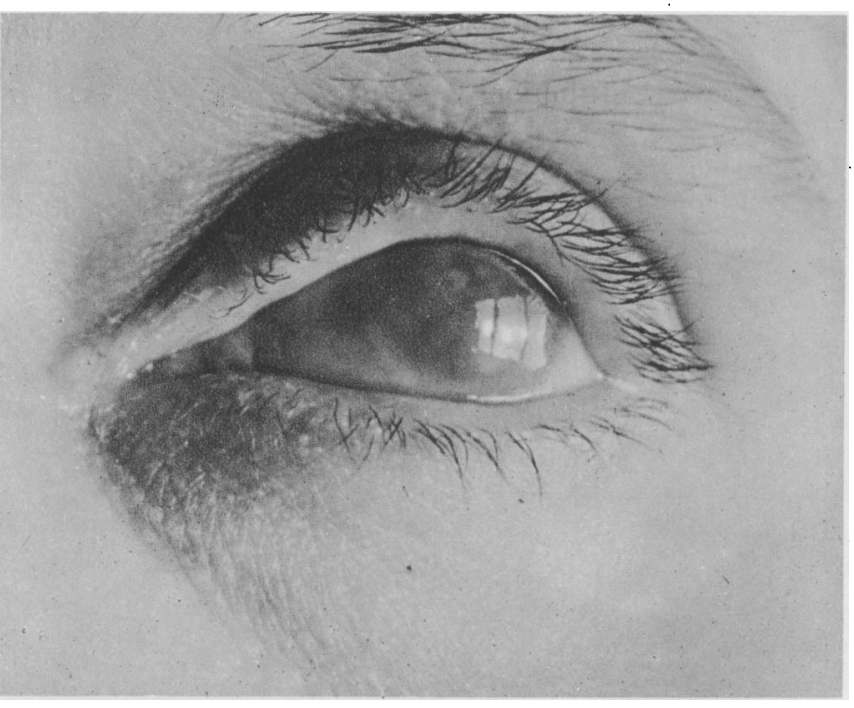

Fia. 1.-Shows the eye before operation. The vacant staring look of a blind eye is apparent. The dark patch above the corneal centre is not the pupil, although it may appear to simulate it.

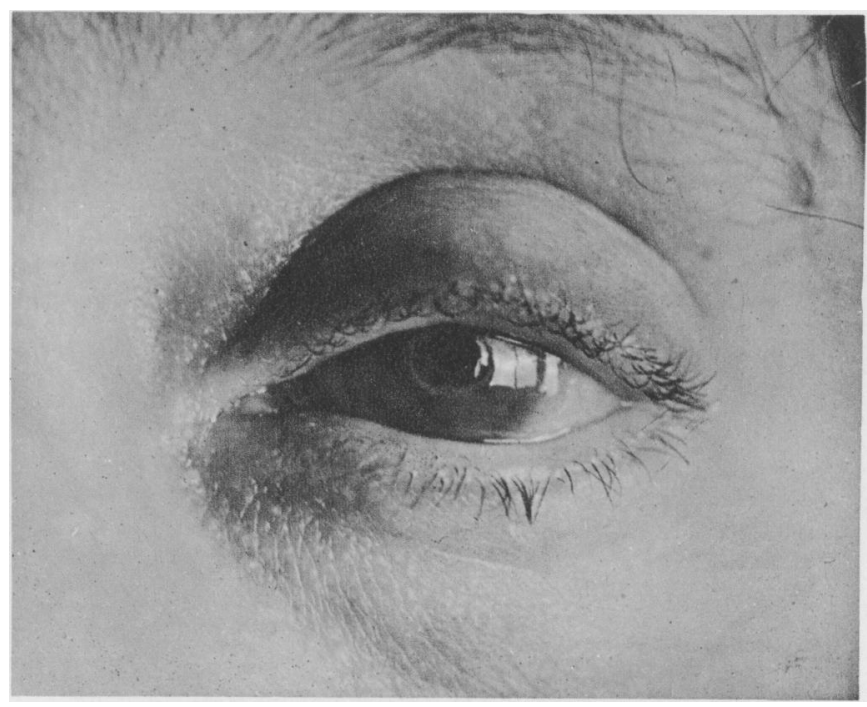

Fia. 2-- Shows the grafted eye twenty-four days after operation. Fixation is now present. The clear dark central gap is seen surrounded by a shelf, which in this case was too excessive. Absence of vascularity
und reaction should be noted. 